\title{
Candida albicans keratitis in an immunocompromised patient
}

This article was published in the following Dove Press journal:

Clinical Ophthalmology

14 October 2010

Number of times this article has been viewed

H Mohammed J Hassan'

Theocharis Papanikolaou ${ }^{2}$

Georgios Mariatos'

Amany Hammad ${ }^{3}$

Hala Hassan ${ }^{4}$

'Ophthalmology Department,

Barnsley Hospital NHS Foundation

Trust, South Yorkshire, England,

UK; ' ${ }^{2}$ phthalmology Department,

Cambridge University Hospitals

NHS Foundation Trust, England,

UK; ${ }^{3}$ Ophthalmology Department,

Rotherham Hospital NHS Foundation

Trust, England, UK; ${ }^{4}$ Corneal and

External Disease Service, Moorfields

Eye Hospital NHS Foundation Trust,

London, England, UK
Correspondence: H Mohammed J Hassan Ophthalmology Department, Barnsley Hospital NHS Foundation Trust, South Yorkshire, England

S75 2EP, UK

Tel +44 I 226432009

Fax +44 I226 432149

Email mohammed.hassan2@nhs.net
Purpose: When investigating a case of unexplained corneal ulceration, we need to think of fungal infection and any predisposing factors.

Methods: A case study of a corneal ulceration in a patient who was HIV positive with a devastating visual outcome.

Results: Therapeutic corneal graft was necessary due to corneal perforation. Immunocompromised state of patient was retrospectively diagnosed.

Conclusions: Candida albicans keratitis is an opportunistic infection of a compromised cornea, and sometimes unknowingly compromised host, which can be initially misdiagnosed. Despite intensive antifungal therapy, occasionally patients require corneal grafting to improve vision, and before it is possible to establish an accurate diagnosis.

Keywords: fungal keratitis, corneal perforation, keratoplasty, human immunodeficiency virus, HIV

\section{Introduction}

Fungal keratitis was first described by Leber in 1879. It is an uncommon cause of corneal infection. If undiagnosed or misdiagnosed, it can lead to devastating visual consequences. The reason we decided to publish this case report is to show the potential blindness from corneal injury in an immunocompromised patient.

The incidence of fungal keratitis varies according to geographical location and ranges from $2 \%$ of keratitis in New York to $35 \%$ in Florida. ${ }^{1}$ Fusarium species are the most common cause of fungal infection in sorthern US (45\%-76\% of fungal keratitis) while Candida and Aspergillus species are most common in Northern US. It must be noted that North East US and the UK are considered temperate regions with similar climates. In a study at the New York Eye and Ear Infirmary, ${ }^{2} 21 \%$ of patients with fungal keratitis were found to be HIV positive. In the UK, in a study by Tuft and Tullo $^{3}$ in the period 2003-2005, only one patient was known to be HIV positive, and in another retrospective study by Galarreta et $\mathrm{al}^{4}$ over a 13 -year period, there were no patients with fungal keratitis in their series known to be HIV positive.

In Africa, a study at Muhimbili Medical Centre ${ }^{5}$ compared the prevalence of HIV infection between patients with fungal keratitis and those with nonfungal keratitis; $81.2 \%$ of cases with fungal keratitis were found to be HIV positive, while only $33 \%$ of those with nonfungal keratitis were HIV positive.

Risk factors for fungal keratitis are young male, healthy, no significant ocular disease, and previous history of trauma (vegetable matter) in agricultural occupations and contact lens users. 
Risk factors for Candida keratitis include: older patient, pre-existing ocular disease, exposure keratopathy, foreign body, corneal surgery, chronic keratitis, chronic use of steroids, and immunosuppressive disease. The relative risk for corneal ulceration in one study was 8.8 in HIV-infected individuals compared to non-HIV-infected individuals. ${ }^{6}$

Clinical features of fungal keratitis include a dull white appearance to the cornea with associated epithelial heaping, grey-white infiltrates with feathery margins, rough texture, raised borders, brown pigmentation, and associated endothelial plaques. Satellite lesions may occur around the primary infiltrate, and hypopyon may also be present.

There are numerous diagnostic tests that can be helpful in identifying fungal elements. Of them, culture in sabouraud dextrose agar, blood agar, and thioglycolate broth can grow fungi.

\section{Methods}

A 36-year-old female patient presented to our Eye Casualty Department complaining of severe pain in the right eye and a decreased vision of 24-hour duration. She was generally fit and well with past medical history of depression and bar investigations for multiple cervical lymph node enlargement in 2004. The patient wasn't on any kind of medication at the time. The patient's history was vague, describing getting in contact with a chemical solution (diluted bleach) while cleaning her kitchen surfaces, after rubbing her eyes with the back of her gloves. Immediately following the incident, she irrigated the eyes thoroughly with water for 10 minutes at home. On presenting to the eye clinic, her acuities with glasses were 6/36 right eye, and 6/6 left eye (Snellen's Acuity Chart). Since there was a history of chemical injury, a litmus pH paper was used, but the tear film was neutral. ${ }^{7}$ The right eye was injected, with mucopurulent discharge, and an edematous cornea with central corneal ulcer measuring $1.5 \mathrm{~mm} \times 1 \mathrm{~mm}$ and associated hypopyon.

The patient was admitted to hospital, corneal scrapings were taken, and antibiotic treatment was initiated with ciprofloxacin eye drops $0.3 \%$ hourly and atropine $1 \%$ BD. There was no growth of any microorganisms from the initial corneal scrapings.

For social reasons, the patient took her own discharge and was lost to follow up until she presented to eye casualty 10 days later with deterioration of her symptoms.

At that stage, right eye vision had reduced to hand movements. Corneal ulcer size was $1 \mathrm{~mm}$ in diameter, and there was no hypopyon. On the basis of the previous history of negative corneal scrapings and our assumption of eye exposure to an alkaline agent, the patient was re-admitted and started on prednisolone tablets $40 \mathrm{mg} /$ day $\mathrm{PO}$ and minims dexamethasone $0.1 \%$ hourly, vitamin $\mathrm{C}$ tablets $3 \mathrm{~g}$ daily and guttae vitamin C QDS, guttae gentamicin $0.3 \%$ 2 hourly, guttae atropine sulfate $1 \%$ TDS, and guttae Ilube $5 \%$ QDS. Over the course of the next 3 days the size of the ulcer reduced significantly.

Heavy growth of Candida albicans, sensitive to amphotericin B was shown on corneal scrapings cultures done on the second admission. Treatment was changed and the patient was started on guttae amphotericin B lipidic emulsion hourly, with debridement of the corneal epithelial debris prior to application, along with tablet ketoconazole $200 \mathrm{mg}$ OD.

Over the next 3 days, the size of the ulcer continued to increase and systemic ketoconazole was discontinued and intravenous amphotericin B was initiated. That seemed to control the infection, but unfortunately 4 days later the cornea spontaneously perforated (Figure 1), and a right therapeutic penetrating keratoplasty was performed.

Intra-operatively, the host cornea was adherent to the anterior crystalline lens capsule and during corneal trephination, the lens nucleus was expressed. The soft lens matter was aspirated and the keratoplasty was completed leaving the eye aphakic (Figure 2). The host cornea was sent for microbiology and histopathology, and the results did not reveal any organisms, and therefore topical and systemic amphotericin B was stopped. Ocular B-scan showed no choroidal or retinal detachments. A week later, as the condition of the graft was improving, right eye vision was counting fingers with no improvement with pinhole. The patient was allowed home with guttae chloramphenicol QDS, Ilube 5\%.

The patient was lost to follow ups due to social reasons and we never knew what her visual outcomes were, but it was counting fingers the day before she took her own discharge.

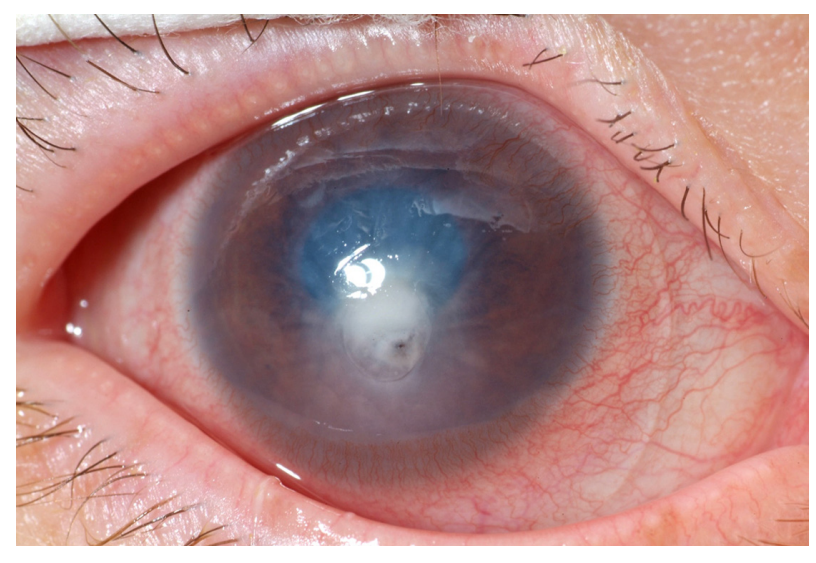

Figure I Perforation of corneal ulcer. 


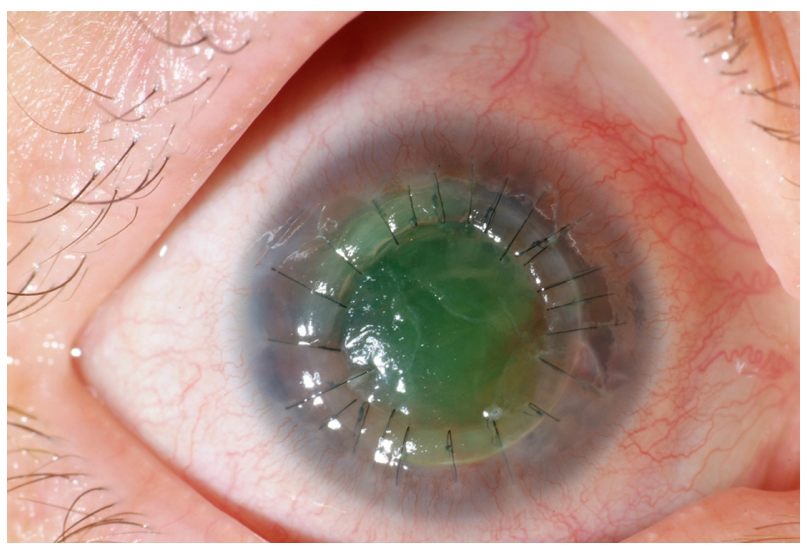

Figure 2 Tectonic/therapeutic corneal graft.

Later on, the patient was contacted by a specialist in genitourinary medicine, requesting her to attend for a sexual health screen. It seems that our patient had had sexual contact with a patient who had recently been diagnosed with HIV a few months before the ocular infection appeared. A full sexual health screen showed a reactive fourth-generation HIV test, ${ }^{7}$ which when repeated confirmed a positive for HIV. This was the patient's first HIV test.

Our patient was expected to undergo immune reconstitution and viral suppression as a response to highly active antiretroviral treatment (HAART), but we were unable to contact her, and we were informed that she was having a difficult time coping socially with her illness.

We have no knowledge whether our patient developed HIV infection before or after the fungal keratitis episode. However due to the low $\mathrm{CD}^{+}$cell count and high viral load, we can assume that she was an HIV patient prior to corneal infection.

\section{Discussion}

According to our literature and Internet search (PubMed, Medscape, and Medline), there was only 1 case, published by Tuft and Tullo, ${ }^{3}$ of fungal keratitis associated with HIV in the last 15 years in the UK. The retrospective study by Moorfields Eye Hospital, ${ }^{4}$ over a period of 13 years revealed no patients in their series known to be HIV positive. However, it appears their HIV status was not checked for.

National HIV testing guidelines published in 2008 recommended increased HIV testing in non-HIV specialties and suggest particular conditions which should raise suspicion of underlying infection. ${ }^{8}$

So is it true that the rate of HIV and Candida albicans keratitis is so low in the UK, or are we simply not looking for them? In Barnsley Hospital, we had one case of
Candida albicans keratitis, and the patient was HIV positive. Retrospectively, we might find the incidence of HIV in patients with Candida albicans keratitis to be higher, if it was screened for.

Candida albicans keratitis is a challenge for ophthalmologists due to its tendency to mimic other conditions. Frequently, cases are misdiagnosed at initial presentation, ${ }^{9}$ resulting in loss of valuable time in treatment. Misdiagnosis may be due to its uncommon occurrence. Further, it is practically impossible to differentiate a bacterial keratitis from Candida albicans keratitis on clinical appearance only, especially in advanced cases. Candida albicans keratitis is an opportunistic infection of an already compromised cornea; thus, the clinical appearance can be deceiving, lacking the trademark corneal lesions features we expect to see. Due to their slow progression and failure to resolve on antibacterial treatment, Candida albicans keratitis is often misdiagnosed as herpetic or amebic.

Corneal scrapings have a major role in misdiagnosis, due to the small amount of corneal material obtained as they are mostly done by junior doctors. If results of cultures are negative but corneal ulcer is persistent, a biopsy should be performed. Microbiological detection of fungal filaments in corneal scrapings can vary from $35 \%$ to $95 \% .{ }^{10}$ A deep corneal biopsy is necessary to obtain tissue for laboratory studies. A significant amount of patients are inadvertently using topical steroid drops by the time diagnosis is established, ${ }^{4}$ with detrimental effect on the prognosis and final visual outcome. This is clearly highlighted in our case, where history of eye exposure to an alkaline agent had led us to the inadvertent use of systemic and topical steroids with an initial favorable response. Since litmus paper $\mathrm{pH}$ was neutral, topical steroids were unnecessary.

The first corneal scraping was negative. When the results from the second culture showed Candida albicans, appropriate amendments were done to the treatment with initiation of systemic and topical amphotericin B lipidic emulsion, and discontinuation of topical and oral steroids.

Amphotericin B is frequently the treatment of choice for Candida infections and related fungi, while miconazole, ketoconazole, itraconazole, and fluconazole can be administered by various routes. ${ }^{11}$ Some fungal infections can be unresponsive to treatment, and according to Lalitha et al, ${ }^{12}$ we can identify risk factors at diagnosis that can serve as prognostic indicators of primary treatment failure in cases of fungal keratitis. Perforation of cornea in cases of fungal keratitis can be a risk factor when an ulcer of $>14 \mathrm{~mm}^{2}$ is present, associated with hypopyon of $>2 \mathrm{~mm}$, and the patient 
is in monotherapy (on topical antifungal drops only). It is not possible to achieve the ocular therapeutic level of antifungal needed by local treatment only. The corneal penetration of all antifungal medications is poor, and even with debridement of the corneal epithelium, the effect is minimal. High concentrations can only be achieved with systemic antifungal treatment.

Unfortunately, when medical therapy fails, surgical intervention is the only solution. About $25 \%$ of fungal keratitis infections will eventually need penetrating keratoplasty. ${ }^{13}$ In our case, perforation of the cornea led to us to do penetrating keratoplasty. Visual prognosis in these cases is often poor especially in drug abuse. ${ }^{14}$ Penetrating keratoplasty is a recognized and effective treatment in cases of severe fungal keratitis unresponsive to antifungal medication. Penetrating keratoplasty is even more beneficial when performed early. ${ }^{15}$

HIV disease in the modern era has very good outcomes, especially if detected early in the course of disease. Interventions using HAART have been shown to provide risk reduction from AIDS and death of up to $97 \% .{ }^{16,17}$ National HIV testing guidelines published in 2008 in the UK recommend increased HIV testing in non-HIV specialties. ${ }^{8}$

In our case, further tests showed an initial CD4 count was $137(\%)$ cells $/ \mathrm{mm}^{3}$ (normal value is between 700 and 1000 cells $/ \mathrm{mm}^{3}$ ), showing significant immunosuppression and a very high viral load of well over 4 million copies $/ \mathrm{mL}$. Genitourinary medicine physicians informed us about her HIV status and how this might affect further management of her corneal disease.

Most common causes of infectious keratitis in HIVpositive patients ${ }^{18}$ are varicella-zoster virus (VZV) and herpes simplex virus (HSV). Both of them can establish latency after primary infection with subsequent reactivation when the immune system of the host is compromised. In VZV, clinical course includes subepithelial infiltrates, stromal keratitis, disciform keratitis, uveitis, and increased intraocular pressure. Varicella keratitis is a self-limited disease. Corneal scarring may occur, but it is rare. Stromal keratitis and uveitis occur in fewer than $10 \%$ of the patients with primary HSV infection. Blepharoconjunctivitis may occur in patients with recurrent ocular HSV infection; it may or may not be associated with epithelial keratitis. Other complications of HSV infection include dendritic and geographic epithelial keratitis, nonnecrotizing stromal keratitis, and iridocyclitis.

Candida species are the most common fungal organisms causing keratitis in HIV-positive patients. Nonfilamentous fungi (Candida) can present in already compromised eyes, while filamentous fungi (Fusarium, Aspergillus) are seen in association with trauma with vegetable matter. Fungal keratitis may be complicated by uveitis, endophthalmitis, and/or retinitis. These complications may cause vitreous abscesses, retinal hemorrhages with or without Roth spots. In severe cases, retinal detachment may develop. Microsporidia is an opportunistic infectious protozoon. These are intracellular parasites capable of causing corneal infection. Superficial keratoconjunctivitis is the most common complication seen in HIV-positive patients infected with microsporidia.

It is believed that the outcome of these ocular infections can be predicted from the $\mathrm{CD}^{+}$cell count. A count of over 200 cells $/ \mathrm{mm}^{3}$ is considered to provide a good prognosis. However, there is active research on this topic at the moment.

HAART $^{19}$ was initiated in the form of Truvada (tenofovir and emtricitabine) and Kaletra (lopinavir/ritonavir). This treatment is ongoing with a positive response. A recent study suggested a survival benefit of more than 13 years in patients with an AIDS-defining illness and over 25 years in those with non-AIDS HIV. ${ }^{20}$

\section{Conclusion}

This case report highlights the fact that Candida albicans external ocular disease associated with HIV can often be overlooked, leading to significant delays in diagnosis and initiation of an effective treatment. While the average interval between presentation to an eye care service and confirmation of the diagnosis of fungal keratitis in the UK is 31 days, the diagnosis in our case took 19 days to confirm. However, we believe that this delay can be even further reduced to be able to improve the visual outcome. Some USA hospitals have a policy to investigate patients with unexplained fungal infections for concomitant immunosuppression, and a similar policy should be introduced in our hospital as well, if not nationally as we advocate.

A number of factors delayed our diagnosis and management of this case. The vague history of trauma or chemical injury given by the patient was one of the factors. The first lesson is that we must never use steroids unless the underlying factors have been identified. When the $\mathrm{pH}$ is normal there is no reason to use steroids.

The second lesson is that if the patient is not improving after a chemical injury, this should alert us for a possible secondary infection of the cornea, which could be of any nature, including fungal. It should also make us think that the immune system of the patient is compromised, such as in HIV. 
The initial corneal scrapings did not show any growth of microorganisms, so the third lesson is to repeat corneal scrapings if there was no clinical improvement within 48 hours.

The fact that the patient took her own discharge made the appropriate management of this case even more difficult. Also, in unusual or difficult to treat cases of corneal ulceration, we need to perform HIV serologic positivity testing. Finally, collaboration with genitourinary specialists is highly recommended when such cases are suspected.

\section{Acknowledgments}

This paper would not have been published without the immense help and contribution of Miss Hala Hassan, BSc (Pharmacology), BSc (Honours), Optometrist Research Fellow, Corneal and External Disease Service, Moorfields Eye Hospital NHS Foundation Trust, London EC1V 2PD, email hala.hassan@moorfields.nhs.uk.

\section{Disclosure}

The authors report no conflicts of interest in this work.

\section{References}

1. Singh D, Verma A. Keratitis, fungal. eMedicine from WebhMD [serial on the Internet]. http://emedicine.medscape.com/article/1194167overview.

2. Ritterband DC, Seedor JA, Shah MK, Koplin RS, McCormick SA. Fungal keratitis at the New York Eye and Ear Infirmary. Cornea 2006;25:264-267.

3. Tuft SJ, Tullo AB. Fungal keratitis in the United Kingdom 2003-2005. Eye. 2009;23:1308-1313.

4. Galarreta DJ, Tuft SJ, Ramsay A, Dart JK. Fungal keratitis in London: microbiological and clinical evaluation. Cornea. 2007;26(9):1082-1086.

5. Mselle J. Fungal keratitis as an indicator of HIV infection in Africa. Trop Doct. 1999;29(3):133-135.
6. Dosa L. HIV is a risk factor for corneal ulceration. Eurotimes Journal [serial on the Internet]. 2003 July. http://www.escrs.org/eurotimes/ July2003/corneal_ulceration.asp

7. Weber B, Fall EH, Berger A, Doerr HW. Reduction of diagnostic window by new fourth-generation human immunodeficiency virus screening assays. J Clin Microbiol. 1998;36(8):2235-2239.

8. British HIV Association. UK National Guidelines for HIV Testing 2008. Available from: http://www.bhiva.org/cms1222621.asp

9. Sun RL, Jones DB, Wilhelmus KR. Clinical characteristics and outcome of Candida keratitis. Am J Ophthalmol. 2007;143(6):1043-1045.

10. Vemuganti GH, Naidu C, Gopinathan U. Rapid detection of fungal filaments in corneal scrapings by microwave heating-assisted Grocott's methenamine silver staining. Indian J Ophthalmol. 2002;50(4): 326-328.

11. Thomas PA. Fungal infections of the cornea. Eye. 2003;17(8): $852-862$.

12. Lalitha P, Prajna NV, Kabra A, Mahadevan K, Srinivasan M. Risk factors for treatment outcome in fungal keratitis. Ophthalmology. 2006;113(4):526-530.

13. Tanure MA, Cohen EJ, Sudesh S, Rapuano CJ, Laibson PR. Spectrum of fungal keratitis at Wills Eye Hospital, Philadelphia, Pennsylvania. Cornea. 2000;19(3):307-312.

14. Hemady RK, Griffin N, Aristimuno B. Recurrent corneal infections in a patient with the acquired immunodeficiency syndrome. Cornea. 1993;12(3):266-269.

15. Xie L, Dong X, Shi W. Treatment of fungal keratitis by penetrating keratoplasty. Br J Ophthalmol. 2001;85(9):1070-1074.

16. Ewings FM, Bhaskaran K, McLean K, Hawkins D, Fisher M; for the UK Register of HIV Seroconverters. Survival following HIV infection of a cohort followed up from seroconversion in the UK. AIDS. 2008;22(1):89-95.

17. Sterne JA, Hernán MA, Ledergerber B, et al; for the Swiss HIV Cohort Study. Long-term effectiveness of potent antiretroviral therapy in preventing AIDS and death: a prospective cohort study. Lancet. 2005;366:378-384.

18. Tandon R, Vajpayee RB, Gupta V, Vajpayee M, Satpathy G, Dada T. Polymicrobial keratitis in an HIV-positive patient. Indian J Ophthalmol. 2003;51(1):87-88

19. Rathbun RC, Liedtke MD, Lockhart SM, Greenfield RA. HIV infection, antiretroviral therapy. eMedicine from WebhMD [serial on the Internet] http://www.emedicine.com/med/topic1533218.htm. Accessed Sep 2, 2009.

20. Walensky RP, Paltiel AD, Losina E, et al. The survival benefit of AIDS treatment in the United States. J Infect Dis. 2006;194:11-19.
Clinical Ophthalmology

\section{Publish your work in this journal}

Clinical Ophthalmology is an international, peer-reviewed journal covering all subspecialties within ophthalmology. Key topics include: Optometry; Visual science; Pharmacology and drug therapy in eye diseases; Basic Sciences; Primary and Secondary eye care; Patient Safety and Quality of Care Improvements. This journal is indexed on Submit your manuscript here: http://www.dovepress.com/clinical-ophthalmology-journal

\section{Dovepress}

PubMed Central and CAS, and is the official journal of The Society of Clinical Ophthalmology (SCO). The manuscript management system is completely online and includes a very quick and fair peer-review system, which is all easy to use. Visit http://www.dovepress.com/ testimonials.php to read real quotes from published authors. 